This is the final peer-reviewed accepted manuscript of:

R. Achilles, M. Manaresi, T. Pruschke, Mixed multiplicities, Segre numbers and Segre classes, Journal of Algebra, Volume 525, 2019, Pages 390-415, ISSN 00218693.

The final published version is available online at: https://doi.org/10.1016/j.jalgebra.2019.01.028

Rights / License:

The terms and conditions for the reuse of this version of the manuscript are specified in the publishing policy. For all terms of use and more information see the publisher's website.

This item was downloaded from IRIS Università di Bologna (https://cris.unibo.it/)

When citing, please refer to the published version. 


\title{
Mixed multiplicities, Segre numbers and Segre classes
}

To the memory of Professor David Rees (1918-2013)

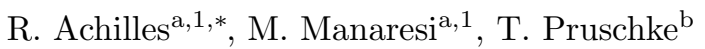 \\ ${ }^{a}$ Dipartimento di Matematica, Università di Bologna, Piazza di Porta S. Donato 5, I-40126 \\ Bologna, Italy \\ ${ }^{b}$ Franz-Kocks-Str. 6, D-33104 Paderborn, Germany
}

\begin{abstract}
Based on classical results of Rees and on multivariate Hilbert polynomials, we define new mixed multiplicities of two arbitrary ideals in a local ring $(A, \mathfrak{m})$ and use them to express the local degrees of all varieties appearing in the GaffneyGassler construction of Segre cycles. We prove that the classical mixed multiplicities of $\mathfrak{m}$ and an arbitrary ideal $I$, which are a special case of the new ones, are equal to the generalized Samuel multiplicities of an ideal in the Rees algebra $R_{I}(A)$. This equality is used to improve a result of Jeffries, Montaño and Varbaro on the degree of the fiber cone of an ideal.

We conclude the paper with formulas (and their inverses) which express the degrees of Segre classes of subschemes of arbitrary projective varieties by generalized Samuel multiplicities or by classical mixed multiplicities. Using the mixed multiplicities of balanced rational normal scrolls, which have been computed by Hoang and Lam, we find the mixed multiplicities of all rational normal scrolls as well as their Segre classes and their generalized Samuel multiplicities.
\end{abstract}

Keywords: Mixed multiplicity, generalized Samuel multiplicity, Segre class, rational normal scroll, fiber cone.

2000 MSC: 13H15 (primary), 14C17, 13A30, 14Q99 (secondary)

\section{Introduction}

The scope of this paper is to express the local degrees of all varieties appearing in the construction of Segre cycles as mixed multiplicities in order to avoid the use of general hypersurfaces. In doing so, a new mixed multiplicity is introduced and studied.

\footnotetext{
* Corresponding author

Email addresses: rudiger.achilles@unibo.it (R. Achilles),

mirella.manaresi@unibo.it (M. Manaresi), thilop@daad-alumni.de (T. Pruschke)

${ }^{1}$ The first and the second author are members of GNSAGA of INdAM.
} 
Local variants of the intersection cycle of Stückrad and Vogel ([34], [13]) were introduced in 1990 by Massey [27], [28] (Lê cycles), in 1995 by Tworzewski [38] and in 1999 by Gaffney and Gassler [15], who defined Segre numbers and polar multiplicities of an ideal in the local ring of a reduced analytic space germ. They used general hyperplanes in the blowup of the germ along the ideal or, alternatively, residual intersections of the polar varieties with hypersurfaces given by general elements of the ideal. In 1997, for ideals in a local ring, Achilles and Manaresi [3] introduced "generalized Samuel multiplicities" as the normalized leading coefficients of a bivariate Hilbert polynomial and applied them to the intersection cycle of Stückrad and Vogel. In the set-ups of Tworzewski and Gaffney-Gassler, the generalized Samuel multiplicities are equal to the extended degree and the Segre numbers, respectively.

In this paper we give the missing description of the local degrees of the intersection of polar varieties with general hypersurfaces as mixed multiplicities. To do this, we introduce new mixed multiplicities $e_{k}(I \mid J, A)$ for an ordered pair $(I, J)$ of arbitrary ideals in a local ring $(A, \mathfrak{m})$. They are defined to be the normalized leading coefficients of a Hilbert polynomial of a trigraded ring. The mixed multiplicities $e_{k}(I \mid \mathfrak{m}, A)$ of the pair $(I, \mathfrak{m})$ are the local multiplicities of the intersection of the polar varieties with a hypersurface defined by a general element from $I$, and the mixed multiplicities $e_{k}(\mathfrak{m} \mid I, A)$ are the polar multiplicities of Gaffney and Gassler [15]. Moreover, the new mixed multiplicity $e_{k}(I \mid \mathfrak{m}, A)$ turns out to be equal to the $j$-multiplicity of the extension of $I$ in the quotient ring of $A$ by $k$ general elements of $\mathfrak{m}$. This result can be seen as an algebraic generalization of Teissier's $\mu^{*}$-sequence [35] of an isolated hypersurface singularity.

When $I$ and $J$ are m-primary, the new multiplicities of $(I, J)$ coincide with the classical mixed multiplicities which are the normalized leading coefficients of the Bhattacharya polynomial [5] and have been defined and studied by Teissier and Risler [35], Katz and Verma [26], Trung [36] and others.

Our main result Theorem 2.4 expresses the degrees of all varieties appearing in the intersection algorithm of [3] as mixed multiplicities and relates them to each other. The constructions of the Lê cycle, the Segre cycle and of the Stückrad-Vogel cycle are special cases. The new mixed multiplicities are needed in the case of Lê and Segre cycles, while for the Stückrad-Vogel cycle the classical mixed multiplicities are sufficient, see Trung [36, Theorem 4.6].

We also prove that the classical mixed multiplicities are equal to the generalized Samuel multiplicities of an ideal in the Rees algebra $R_{J}(A)$, see Theorem 3.1. As an application of this result we can describe the degree of the fiber cone of an ideal in terms of a classical mixed multiplicity, see Theorem 3.3 and Remark 5. This generalizes a recent result of Jeffries, Montaño and Varbaro [25, Theorem 3.1].

We conclude the paper with a collection of explicit formulas which express the degree of Segre classes of subschemes of projective varieties by generalized Samuel multiplicities or classical mixed multiplicities. The first of such formulas has been given by van Gastel [16, Corollary 3.7] in 1991 and they have been used by Achilles and Manaresi in 1997 [3, Corollary 4.3] to give a method for 
the computation of the Segre class. A slightly more general formula has been implemented in REDUCE by Achilles and Aliffi [1] and allows to compute Segre classes in projective varieties with arbitrary singularities. Recently Harris [17] has given another approach via linear projections. Several other algorithms to compute Segre classes of subschemes of projective space have been presented by Aluffi [4], Eklund, Jost and Peterson [11], and Helmer [20].

Using the mixed multiplicities of balanced rational normal scrolls, which have been computed by Hoang and Lam [23], we find the mixed multiplicities of all rational normal scrolls. Then, by the conversion formulas, we obtain the Segre class and the generalized Samuel multiplicities of rational normal scrolls. For the first generalized Samuel multiplicity, the so-called $j$-multiplicity, there was already a formula by Jeffries, Montaño and Varbaro [25, Theorem 3.3].

\section{Hilbert functions of multigraded rings and mixed multiplicities}

In this section we first recall some well-known facts on Hilbert functions and Hilbert polynomials of standard multigraded rings, then we introduce new multiplicities for ordered couples of arbitrary ideals in local rings, which generalize the classical mixed multiplicities.

We shall use multi-index notation: if $\alpha=\left(\alpha_{1}, \ldots, \alpha_{m}\right)$ is an $m$-tuple of non-negative integers, that is, $\alpha \in \mathbb{N}^{m}$, then $|\alpha|=\alpha_{1}+\cdots+\alpha_{m}$ and $\left(\begin{array}{l}\alpha \\ \beta\end{array}\right)=$ $\left(\begin{array}{c}\alpha_{1} \\ \beta_{1}\end{array}\right) \ldots\left(\begin{array}{c}\alpha_{m} \\ \beta_{m}\end{array}\right)$.

In the following, by a standard multigraded ring we mean a ring $S=\bigoplus_{\alpha \in \mathbb{N}^{m}} S_{\alpha}$ such that

(i) $S_{\alpha}$ are additive subgroups,

(ii) $S_{\alpha} \cdot S_{\beta} \subseteq S_{\alpha+\beta}$ for all $\alpha, \beta \in \mathbb{N}^{m}$,

(iii) $S$ is as an $S_{0}$-algebra $(0=(0, \ldots, 0))$ finitely generated by homogeneous elements of total degree one, that is, by all $S_{\alpha}$ with $|\alpha|=1$.

Let $S=\bigoplus_{\alpha \in \mathbb{N}^{m}} S_{\alpha}$ be a standard multigraded ring of (Krull-)dimension $\delta$ and assume that $S_{0}$ is an Artinian ring. The Hilbert function of $S$ is defined to be

$$
h(\alpha)=h_{S}(\alpha)=\operatorname{length}_{S_{0}}\left(S_{\alpha}\right) .
$$

For $\alpha_{1}, \ldots, \alpha_{m}$ sufficiently large, the function $h_{S}(\alpha)$ becomes a polynomial $p_{S}(\alpha)$, the Hilbert polynomial of $S$, which can be written in the form

$$
p_{S}(\alpha)=\sum_{\substack{\beta \in \mathbb{N} m \\
|\beta| \leq \delta-m}} a_{\beta}\left(\begin{array}{c}
\alpha \\
\beta
\end{array}\right)
$$

with $a_{\beta} \in \mathbb{Z}$. Of special importance are the coefficients $a_{\beta}$ with $|\beta|=\delta-m$, which are all non-negative (see [42, Thm. 7, p. 757 and Thm. 11, p. 759], [5] for the bigraded case and [21, Thm. 4.1] for the $m$-graded case) but could be all zero (see Trung [36] for results on their positivity). Despite of this fact we shall call them the normalized leading coefficients of $p_{S}$. 
Also the so-called sum transforms of $h$ become polynomials with integer coefficients. For example, the sum transform of $h$ with respect to the first variable is defined to be the function $g(\alpha)=\sum_{u=0}^{\alpha_{1}} h\left(u, \alpha_{2}, \ldots, \alpha_{m}\right)$, which for $\alpha_{1}, \ldots, \alpha_{m}$ sufficiently large can be written as

$$
\sum_{\substack{\beta \in \mathbb{N}^{m} \\
|\beta| \leq \delta-m+1}} b_{\beta}\left(\begin{array}{l}
\alpha \\
\beta
\end{array}\right)
$$

with $b_{\beta} \in \mathbb{Z}$. If $|\beta|=\delta-m+1$, then $b_{\beta} \geq 0$ and if furthermore $\beta_{1} \geq 1$, then $b_{\beta}=a_{\left(\beta_{1}-1, \beta_{2}, \ldots, \beta_{m}\right)}$. We observe that $b_{\left(0, \beta_{2}, \ldots, \beta_{m}\right)}, \beta_{2}+\cdots+\beta_{m}=\delta-m+1$, is a new leading coefficient.

Let $A$ be a $d$-dimensional Noetherian local ring with unique maximal ideal $\mathfrak{m})$ or a standard graded algebra such that $\left(A_{0}, \mathfrak{n}\right)$ is an Artinian local ring and $\mathfrak{m}=\left(\mathfrak{n}, A_{1}\right)$ is the unique homogeneous maximal ideal of $A$. For an ideal $I \subset A$ we denote by

$$
R=R_{I}(A)=A[I t] \subset A[t] \quad(t \text { is an indeterminate })
$$

the Rees algebra of $I$ and by

$$
G=G_{I}(A)=\bigoplus_{i \geq 0} I^{i} / I^{i+1}
$$

the associated graded ring of $A$ with respect to $I$. We remember that $\operatorname{dim} G=d$ while $\operatorname{dim} R=d+1$ if there exists a $d$-dimensional prime ideal $\mathfrak{p} \subset A$ such that $I \nsubseteq \mathfrak{p}$ and $\operatorname{dim} R=d$ otherwise, see, for example [22, Thm. 9.7, p. 51].

For ideals $I, J \subset A$ we set

$$
I:\langle J\rangle:=\left\{a \in A \mid \text { there is a positive integer } n \text { such that } a \cdot J^{n} \subseteq I\right\} .
$$

Then $\operatorname{dim} R_{I}(A)=\operatorname{dim} A$ if and only if $\operatorname{dim} A /(0:\langle I\rangle)<\operatorname{dim} A$.

Now, in order to define mixed multiplicities $e_{i}(I \mid J)(i=0, \ldots, d-1)$ for arbitrary proper ideals $I, J \subset A$, we consider the trigraded ring

$$
S=S(I, J ; A)=\bigoplus_{k, i, j \geq 0} S_{(k, i, j)}=G_{\mathfrak{m}}\left(G_{I}\left(R_{J}(A)\right)\right)=\bigoplus_{k, i, j \geq 0} \frac{\mathfrak{m}^{k} I^{i} J^{j}+I^{i+1} J^{j}}{\mathfrak{m}^{k+1} I^{i} J^{j}+I^{i+1} J^{j}}
$$

whose dimension is $d+1$ if there exist a $d$-dimensional prime ideal $\mathfrak{p} \subset A$ such that $J \nsubseteq \mathfrak{p}$ and $d$ otherwise.

The sum transform of the Hilbert function $h_{S}(k, i, j)=\operatorname{length}\left(S_{(k, i, j)}\right)$ of $S$ with respect to $k$ is

$$
h_{S}^{(1,0,0)}(k, i, j)=\operatorname{length}\left(\frac{I^{i} J^{j}}{\mathfrak{m}^{k+1} I^{i} J^{j}+I^{i+1} J^{j}}\right),
$$

which for $k, i, j$ sufficiently large becomes a polynomial of the form

$$
\sum_{\substack{\ell, m, n \geq 0 \\
\ell+m+n \leq d-1}} a_{(\ell, m, n)}(I, J ; A)\left(\begin{array}{c}
k \\
\ell
\end{array}\right)\left(\begin{array}{c}
i \\
m
\end{array}\right)\left(\begin{array}{l}
j \\
n
\end{array}\right),
$$

where $a_{(\ell, m, n)}(I, J ; A)$ are non-negative integers, possibly all equal to zero. 
Definition 1. The mixed multiplicities of the ordered pair $(I, J)$ of ideals $I, J \subset$ $A$ are defined to be

$$
e_{i}(I \mid J)=e_{i}(I \mid J, A)=a_{(0, i, d-1-i)}(I, J ; A), \quad i=0, \ldots, d-1 .
$$

Remark 1. If both $I$ and $J$ are m-primary ideals, then $e_{i}(I \mid J)(i=0, \ldots, d-1)$ are the first $d$ normalized leading coefficients of the Bhattacharya polynomial [5] and if only $I$ is $\mathfrak{m}$-primary and $J$ has positive height, then the $e_{i}(I \mid J)$ are the mixed multiplicities introduced and studied by Katz and Verma, see [26], [39], [40]. In fact, if $I$ is $\mathfrak{m}$-primary, then for sufficiently large $k$ we have

$$
\frac{I^{i} J^{j}}{\mathfrak{m}^{k+1} I^{i} J^{j}+I^{i+1} J^{j}}=\frac{I^{i} J^{j}}{I^{i+1} J^{j}}
$$

and the length of the latter term was used by Katz and Verma to define $e_{i}(I \mid J)$.

Remark 2. If $\operatorname{dim} R_{J}(A)=d:=\operatorname{dim} A$, or equivalently, if for all $d$-dimensional prime ideals $\mathfrak{p} \subset A$ one has $J \subseteq \mathfrak{p}$, that is, $\operatorname{dim} A /(0:\langle J\rangle)<\operatorname{dim} A$, then the degree of the Hilbert polynomial (1) is strictly smaller than $d-1$. Hence by Definition 1 for all $i=0, \ldots, d-1$ it holds $e_{i}(I \mid J)=0$.

Trung's mixed multiplicities ([36], p. 34) are different from ours since they are defined using the actual degree of the Hilbert polynomial and under the hypothesis that $I$ is $\mathfrak{m}$-primary. Under this hypothesis, they coincide with ours if $\operatorname{dim} R_{J}(A)=\operatorname{dim} A+1$, that is, $\operatorname{dim} A=\operatorname{dim} A /(0:\langle J\rangle)$ (see also [36], Lemma 3.1).

Remark 3. We can define $e_{d}(I \mid J, A)$ using the sum transform $h_{S}^{(1,1,0)}$ of the function $h_{S}^{(1,0,0)}(k, i, j)$ with respect to the variable $i$ and it turns out that

$$
e_{d}(I \mid J, A)=0 .
$$

In fact, by contradiction, assume $e_{d} \neq 0$. Fix a sufficiently large integer $k$ and set

$$
\tilde{S}_{(i, j)}=I^{i} J^{j} /\left(\mathfrak{m}^{k+1} I^{i} J^{j}+I^{i+1} J^{j}\right) \text { and } \tilde{S}=\bigoplus_{i, j \geq 0} \tilde{S}_{(i, j)} .
$$

The assumption $e_{d} \neq 0$ is equivalent to the existence of a highest dimensional prime ideal $\mathfrak{p}$ of $\tilde{S}$, such that $\tilde{S}_{(1,0)} \subseteq \mathfrak{p}$ (cf. e.g. $\left.[3,1.2,1.5]\right)$. If $\tilde{S}_{(1,0)} \subseteq \mathfrak{p}$ then

$$
\begin{aligned}
\operatorname{dim} \tilde{S}=\operatorname{dim} \tilde{S} / \mathfrak{p} & \leq \operatorname{dim} \tilde{S} / \tilde{S}_{(1,0)} \tilde{S}= \\
& =\operatorname{dim} \bigoplus_{j \geq 0} \frac{J^{j}}{\mathfrak{m}^{k+1} J^{j}+I J^{j}} \leq \operatorname{dim} \bigoplus_{j \geq 0} \frac{J^{j}}{\mathfrak{m}^{k+1} J^{j}}=s(J) \leq d,
\end{aligned}
$$

where $s(J)$ denotes the analytic spread of $J$. From $\operatorname{dim} \tilde{S} \leq d$ and $h_{\tilde{S}}^{(1,0)}(i, j)=$ $h_{S}^{(1,1,0)}(k, i, j)$ it follows that $h_{S}^{(1,1,0)}$ becomes a polynomial of degree at most $d-1$, for large $i, j$. So $e_{l}=0$, for all $l=0, \ldots, d$, which contradicts $e_{d} \neq 0$. 
Next we recall the definition of the generalized Samuel multiplicities of an ideal $I \subset A$ from [3]. We consider the bigraded ring

$$
T=\bigoplus_{i, j \geq 0} T_{(i, j)}=G_{\mathfrak{m}}\left(G_{I}(A)\right)=\bigoplus_{i, j \geq 0} \frac{\mathfrak{m}^{i} I^{j}+I^{j+1}}{\mathfrak{m}^{i+1} I^{j}+I^{j+1}}
$$

The double sum transform of the Hilbert function $h_{T}(i, j)=\operatorname{length}\left(T_{(i, j)}\right)$ of $T$ with respect to $i$ and $j$ for sufficiently large $i, j$ becomes a polynomial of the form

$$
\sum_{\substack{m, n \geq 0 \\
|m+n| \leq d}} a_{(m, n)}(\mathfrak{m}, I ; A)\left(\begin{array}{c}
i \\
m
\end{array}\right)\left(\begin{array}{l}
j \\
n
\end{array}\right)
$$

where $a_{(m, n)}(\mathfrak{m}, I ; A)$ are non-negative integers, at least one of them positive.

Definition 2 (Achilles-Manaresi [2], [3]). The generalized Samuel multiplicities of the ideal $I$ are defined to be

$$
c_{i}(I)=c_{i}(I, A)=a_{(i, d-i)}(\mathfrak{m}, I ; A), \quad i=0, \ldots, d .
$$

The $j$-multiplicity $j(I)$ of the ideal $I$ introduced in [2] is the non-negative integer $c_{0}(I, A)$.

We remark that if the ideal $I$ is $\mathfrak{m}$-primary then $c_{0}(I)=c_{0}(I, A)=e(I, A)$, the Samuel multiplicity of $I$. In the case $I=\mathfrak{m}$ we shall simply write $e(A)$. If moreover $A$ is a standard graded algebra such that $A_{0}$ is a local ring, then $e(A)$ is the multiplicity or degree of $A$.

Intersection algorithm. The generalized Samuel multiplicities of $I$ can be computed by an algorithm mimicking the intersection algorithm of Stückrad and Vogel [41]. This algorithm needs "generic elements" of the ideal $I$ and produces at the same time the generalized Samuel multiplicities $c_{i}(I, A)$ and the mixed multiplicities $e_{i}(\mathfrak{m} \mid I)$ and $e_{i}(I \mid \mathfrak{m})$.

In order to have "generic elements" of $I=\left(\xi_{1}, \ldots, \xi_{n}\right) A$ in the sense of $[12$, $3.3]$ or $[13,1.5 .13]$, we extend $A$ by $n^{2}$ new indeterminates $U:=\left\{U_{i j} \mid 1 \leq i, j \leq\right.$ $n\}$ and pass to the localization $B=A[U]_{\mathfrak{m} A[U]}$. Then, by Cramer's rule, the generic elements

$$
x_{i}:=\sum_{j=1}^{n} U_{i j} \xi_{j} \in I B, \quad 1 \leq i \leq n
$$

generate $J:=I B$. Following [13], we call $\underline{x}=\left(x_{1}, \ldots, x_{n}\right)$ a transformed set of generators for $I B$. By the genericity of $\underline{x}$, every subset of $s=s(I)=$ $s(J)$ elements of $\left\{x_{1}, \ldots, x_{n}\right\}$ forms a minimal reduction of $J$, in particular $\sqrt{\left(x_{1}, \ldots, x_{s}\right)}=\sqrt{J}$. We remark that the ring extension $A \rightarrow B$ is faithfully flat and $\operatorname{dim} A=\operatorname{dim} B$ (see, for example [13, 1.5.14]). In the case $A$ is an algebra one has to consider the extension

$$
A \rightarrow A_{\mathfrak{m}} \rightarrow B=A_{\mathfrak{m}}[U]_{\mathfrak{m} A_{\mathfrak{m}}[U]}
$$


[8, Proposition 1.5.15].

We define a cycle $v(\underline{x}, B)$ of $B$ supported on $V(I B)=V(\underline{x} B)$ by the following intersection algorithm in $B$. Set $\mathfrak{b}_{-1}:=(0), x_{0}:=0, J:=\underline{x} B$, and inductively

$$
\mathfrak{b}_{k}:=\left(\mathfrak{b}_{k-1}+x_{k} B\right):_{B}\langle J\rangle:=\bigcup_{n \geq 0}\left(\left(\mathfrak{b}_{k-1}+x_{k} B\right):_{B} J^{n}\right) \quad(0 \leq k \leq s) .
$$

Observe that $\mathfrak{b}_{s}=B$. Then

$$
v^{k}(\underline{x}, B):=\sum_{\mathfrak{p}} \operatorname{length}\left(B /\left(\mathfrak{b}_{k-1}+x_{k} B\right)\right)_{\mathfrak{p}}[\mathfrak{p}],
$$

where the sum is taken over all $(d-k)$-dimensional associated prime ideals $\mathfrak{p}$ of $B /\left(\mathfrak{b}_{k-1}+x_{k} B\right)$ that contain $J$ and $[\mathfrak{p}]$ denotes the cycle associated with $\mathfrak{p}$. We define

$$
v(\underline{x}, B):=\sum_{k=0}^{s} v^{k}(\underline{x}, B),
$$

and the degree of $v^{k}(\underline{x}, B)$ by

$$
\operatorname{deg} v^{k}(\underline{x}, B):=\sum_{\mathfrak{p}} \operatorname{length}\left(B /\left(\mathfrak{b}_{k-1}+x_{k} B\right)\right)_{\mathfrak{p}} \cdot e(B / \mathfrak{p}) .
$$

By [3, Theorem 4.1] we have

$$
c_{k}(I, A)=c_{k}(J, B)=\operatorname{deg} v^{d-k}(\underline{x}, B), \quad k=0, \ldots, s
$$

and $c_{k}(I, A)=c_{k}(J, B)=0$ for $k=s+1, \ldots, d$.

We shall prove that the intersection algorithm produces not only the generalized Samuel multiplicities $c_{k}(I, A)$ but also the mixed multiplicities $e_{d-k}(I \mid \mathfrak{m})$ and $e_{k}(\mathfrak{m} \mid I)$. In order to prove this, we need to know how these numbers behave under the two operations of the intersection algorithm, that is, under factorization of generic elements and under removing certain components of the zero ideal of the ring. We begin with a lemma due to D. Rees, which is essentially [33, Lemma 1.2].

Lemma 2.1 (D. Rees). Let $A$ be a local ring or a standard graded algebra such that $A / \mathfrak{m}$ is infinite and let $I, J$ be ideals of $A$. If $\Sigma$ is a finite set of ideals of $A$ not containing $I$, then there exists an element $x$ of $I$ not contained in any of the ideals in $\Sigma$ such that for all large $i$ and for all $j \geq 0$ we have

$$
x A \cap I^{i} J^{j}=x I^{i-1} J^{j} .
$$

Proof. The lemma is a variation of [33, Lemma 1.2]. Note that the assumptions about ideals being prime are not needed since the residue field is infinite. One uses the well-known fact that a vector space over such a field cannot be the union of a finite number of proper linear subspaces. Then Rees' proof still works. 
Lemma 2.2. Let $A$ be a local ring or a standard graded algebra of dimensiond such that $A / \mathfrak{m}$ is infinite and let $I, J$ be ideals of $A$ which are not contained in any associated $d$-dimensional prime ideal of $A$. Then there are elements $x \in I$ and $y \in J$ such that for $l=0, \ldots, d-2$ it holds

$$
e_{l}(I \mid J, A / x A)=e_{l}(I \mid J, A) \quad \text { and } \quad e_{l}(I \mid J, A / y A)=e_{l+1}(I \mid J, A) .
$$

The elements $x$ and $y$ can be chosen outside a finite number of proper ideals of $A$ which do not contain I or $J$, respectively.

Proof. Let $\Sigma$ be the set composed of the finite number of ideals in the lemma, of all associated $d$-dimensional prime ideals of $A$ and of all ideals properly contained in $I$ which correspond to the contraction of a relevant associated prime ideal of $\tilde{S}$ (as defined by (2) in Remark 3) to $\tilde{S}_{(1,0)}=I /\left(\mathfrak{m}^{k+1} I+I^{2}\right)$. Then by Lemma 2.1 there is an element $x \in I$ not contained in any of the ideals in $\Sigma$ such that for all large $i$ we have $x A \cap I^{i} J^{j}=x I^{i-1} J^{j}$ and $\operatorname{dim}(A / x A)=d-1$. As in Remark 3, denote by $\tilde{S}$ and $\tilde{S}(A / x A)$ the bigraded rings whose Hilbert polynomials define $e_{l}(I \mid J, A)$ and $e_{l}(I \mid J, A / x A)$ respectively. We obtain

$$
\begin{aligned}
{[\tilde{S}(A / x A)]_{(i, j)} } & =\frac{I^{i} J^{j}+x A}{\mathfrak{m}^{k+1} I^{i} J^{j}+I^{i+1} J^{j}+x A} \\
& \cong \frac{I^{i} J^{j}}{I^{i} J^{j} \cap\left(\mathfrak{m}^{k+1} I^{i} J^{j}+I^{i+1} J^{j}+x A\right)} \\
& =\frac{I^{i} J^{j}}{\mathfrak{m}^{k+1} I^{i} J^{j}+I^{i+1} J^{j}+\left(x A \cap I^{i} J^{j}\right)} \\
& =\frac{I^{i} J^{j}}{\mathfrak{m}^{k+1} I^{i} J^{j}+I^{i+1} J^{j}+x I^{i-1} J^{j}}=[\tilde{S} / x \tilde{S}]_{(i, j)} .
\end{aligned}
$$

This shows that $e_{0}(I \mid J, A / x A), \ldots, e_{d-2}(I \mid J, A / x A)$ are equal to the normalized leading coefficients of the Hilbert polynomial of $\tilde{S} / x \tilde{S}$. For $i$ and $j$ sufficiently large, taking into account that $x$ avoids every relevant homogeneous associated prime ideal of $\tilde{S}$, we can compute the top degree of the Hilbert polynomial of $\tilde{S} / x \tilde{S}$ as follows:

$$
\begin{aligned}
& \text { length }[\tilde{S} / x \tilde{S}]_{(i, j)}=\text { length } \tilde{S}_{(i, j)}-\text { length } \tilde{S}_{(i-1, j)} \\
= & \sum_{l=0}^{d-1} e_{l}(I \mid J)\left[\left(\begin{array}{c}
i \\
d-1-l
\end{array}\right)-\left(\begin{array}{c}
i-1 \\
d-1-l
\end{array}\right)\right] \cdot\left(\begin{array}{l}
j \\
l
\end{array}\right)+\text { terms of lower degree } \\
= & \sum_{l=0}^{d-2} e_{l}(I \mid J)\left(\begin{array}{c}
i \\
d-2-l
\end{array}\right)\left(\begin{array}{l}
j \\
l
\end{array}\right)+\text { terms of lower degree. }
\end{aligned}
$$

This proves the first statement of the lemma.

The proof of the second statement is similar. This time Lemma 2.1 is applied to the set $\Sigma$ that is composed of all associated $d$-dimensional prime ideals of $A$ and of all ideals properly contained in $J$ which correspond to the contraction of 
a relevant associated prime ideal of $\tilde{S}$ to $\tilde{S}_{(0,1)}=J /\left(\mathfrak{m}^{k+1} J+I J\right)$. Then there is an element $y \in J$ such that for all large $j$ we have $y A \cap I^{i} J^{j}=y I^{i} J^{j-1}$, $\operatorname{dim}(A / y A)=d-1$, and we obtain

$$
\begin{aligned}
{[\tilde{S}(A / y A)]_{(i, j)} } & =\frac{I^{i} J^{j}+y A}{\mathfrak{m}^{k+1} I^{i} J^{j}+I^{i+1} J^{j}+y A} \\
& \cong \frac{I^{i} J^{j}}{I^{i} J^{j} \cap\left(\mathfrak{m}^{k+1} I^{i} J^{j}+I^{i+1} J^{j}+y A\right)} \\
& =\frac{I^{i} J^{j}}{\mathfrak{m}^{k+1} I^{i} J^{j}+I^{i+1} J^{j}+\left(y A \cap I^{i} J^{j}\right)} \\
& =\frac{I^{i} J^{j}}{\mathfrak{m}^{k+1} I^{i} J^{j}+I^{i+1} J^{j}+y I^{i} J^{j-1}}=[\tilde{S} / y \tilde{S}]_{(i, j)} .
\end{aligned}
$$

This shows that $e_{0}(I \mid J, A / y A), \ldots, e_{d-2}(I \mid J, A / y A)$ are equal to the normalized leading coefficients of the Hilbert polynomial of $\tilde{S} / y \tilde{S}$. Since $y$ avoids every relevant homogeneous associated prime ideal of $\tilde{S}$, for $i$ and $j$ sufficiently large, we have

$$
\begin{gathered}
\text { length }[\tilde{S} / y \tilde{S}]_{(i, j)}=\operatorname{length} \tilde{S}_{(i, j)}-\operatorname{length} \tilde{S}_{(i, j-1)} \\
=\sum_{l=0}^{d-1} e_{l}(I \mid J)\left(\begin{array}{c}
i \\
d-1-l
\end{array}\right) \cdot\left[\left(\begin{array}{l}
j \\
l
\end{array}\right)-\left(\begin{array}{c}
j-1 \\
l
\end{array}\right)\right]+\text { terms of lower degree } \\
=\sum_{l=1}^{d-1} e_{l}(I \mid J)\left(\begin{array}{c}
i \\
d-1-l
\end{array}\right)\left(\begin{array}{c}
j \\
l-1
\end{array}\right)+\text { terms of lower degree, }
\end{gathered}
$$

which proves the lemma.

The next lemma shows that in order to compute mixed multiplicities, certain components of the zero ideal of the ring can be removed.

Lemma 2.3. Let $I, J$ be ideals of $A$ and assume that $\operatorname{dim} A=\operatorname{dim}(A / 0$ : $\langle J\rangle) \geq 1$. Then, for all $l=0, \ldots, d-1$, we have

$$
e_{l}(I \mid J)=e_{l}(I \mid J, A / 0:\langle J\rangle) .
$$

Similarly, if $\operatorname{dim} A=\operatorname{dim}(A / 0:\langle I\rangle) \geq 1$, then for all $l=0, \ldots, d-1$, we have

$$
e_{l}(I \mid J)=e_{l}(I \mid J, A / 0:\langle I\rangle) .
$$

Proof. We have for all $i, j \geq 0$ and $k$ large but fixed

$$
\begin{aligned}
\frac{I^{i} J^{j}+0:\langle J\rangle}{\mathfrak{m}^{k+1} I^{i} J^{j}+I^{i+1} J^{j}+0:\langle J\rangle} & \cong \frac{I^{i} J^{j}}{I^{i} J^{j} \cap\left(\mathfrak{m}^{k+1} I^{i} J^{j}+I^{i+1} J^{j}+0:\langle J\rangle\right)} \\
& =\frac{I^{i} J^{j}}{\mathfrak{m}^{k+1} I^{i} J^{j}+I^{i+1} J^{j}+\left(I^{i} J^{j} \cap(0:\langle J\rangle)\right)} .
\end{aligned}
$$


By the Artin-Rees Lemma, there is an integer $c$ such that for all large $j$ we have

$$
I^{i} J^{j} \cap(0:\langle J\rangle)=J^{j-c}\left(I^{i} J^{c} \cap(0:\langle J\rangle)\right) \subseteq J^{j-c}(0:\langle J\rangle)=0
$$

so that the term of (5) is isomorphic to

$$
\frac{I^{i} J^{j}}{\mathfrak{m}^{k+1} I^{i} J^{j}+I^{i+1} J^{j}} .
$$

If we take the lengths over these isomorphisms, we obtain that the Hilbert functions defining $e_{l}(I \mid J)$ and $e_{l}(I \mid J, A / 0:\langle J\rangle)$, respectively, coincide for large $i, j$. Hence, their Hilbert polynomials coincide.

The proof of the second statement is similar.

Now we are ready to express the mixed multiplicities $e_{d-k}(I \mid \mathfrak{m})$ and $e_{k}(\mathfrak{m} \mid I)$ by Samuel multiplicities from the intersection algorithm. We shall prove that $e_{d-k}(I \mid \mathfrak{m})$ is the degree of the $k$ th intersection and $e_{k}(\mathfrak{m} \mid I)$ that of the $k$ th residual intersection.

Theorem 2.4. With the previous notations, assume that $d=\operatorname{dim} A \geq 1$. Then

(i) for $k=1, \ldots, s$,

(a) if $\operatorname{dim}\left(B / \mathfrak{b}_{k-1}\right)=d-k+1$, then

$$
e_{d-k}(I \mid \mathfrak{m}, A)=e_{d-k}(I B \mid \mathfrak{m} B, B)=e\left(B /\left(\mathfrak{b}_{k-1}+x_{k} B\right)\right)
$$

and

$$
e_{k-1}(\mathfrak{m} \mid I, A)=e_{k-1}(\mathfrak{m} B \mid I B, B)=e\left(B / \mathfrak{b}_{k-1}\right) .
$$

(b) if $\operatorname{dim}\left(B / \mathfrak{b}_{k-1}\right)<d-k+1$, then

$$
e_{d-k}(I \mid \mathfrak{m}, A)=0 \quad \text { and } \quad e_{k-1}(\mathfrak{m} \mid I, A)=0 .
$$

(c)

$$
c_{d-k}(I, A)=e_{d-k}(I \mid \mathfrak{m}, A)-e_{k}(\mathfrak{m} \mid I, A) ;
$$

(ii) for $k=s+1, \ldots, d$

$$
e_{d-k}(I \mid \mathfrak{m}, A)=e_{k-1}(\mathfrak{m} \mid I, A)=c_{d-k}(I, A)=0 ;
$$

(iii) for $k=1, \ldots, d-\operatorname{dim}(A / I)-1$

$$
e_{d-k}(I \mid \mathfrak{m}, A)=e_{k}(\mathfrak{m} \mid I, A) \quad \text { and } \quad c_{d-k}(I, A)=0 .
$$

Proof. Since the faithfully flat ring extension $A \rightarrow B$ does not affect the Hilbert functions, it is sufficient to prove the assertions regarding the ring $B$. Once (i) and (ii) have been proved, (iii) is an immediate consequence of the intersection algorithm.

We shall prove (i) and (ii) by induction on $d=\operatorname{dim} A$. 
Let us consider the case $d=1$. Then the analytic spread $s$ of $I$ is either 0 or 1 . If $s=0$, then $I$ is nilpotent and $\operatorname{dim} R_{I}(A)=\operatorname{dim} A=1$, hence $e_{0}(I \mid \mathfrak{m}, A)=e_{0}(\mathfrak{m} \mid I, A)=0$. It is clear that also $c_{0}(I)=0$. If $s=1$, then $I$ is not nilpotent and $\operatorname{dim} R_{I}(A)=\operatorname{dim} A+1=2$. By Lemma 2.3 and [26, Lemma 2.2] or [36, Lemma 3.1],

$$
e_{0}(I B \mid \mathfrak{m} B, B)=e_{0}(I B \mid \mathfrak{m} B, B / 0:\langle I B\rangle)=e(I B, B / 0:\langle I B\rangle)
$$

and, since $x_{1}$ is a generic element of $I B$ and $B / 0:\langle I B)$ is Cohen-Macaulay,

$$
\begin{aligned}
e(I B, B / 0:\langle I B\rangle) & =e\left(x_{1} B, B / 0:\langle I B\rangle\right)=\operatorname{length}\left(B /\left(0:\langle I B\rangle+x_{1} B\right)\right) \\
& =e\left(B /\left(0:\langle I B\rangle+x_{1} B\right)\right) .
\end{aligned}
$$

By (6) and (7),

$$
e_{0}(I B \mid \mathfrak{m} B, B)=e\left(B /\left(0:\langle I B\rangle+x_{1} B\right)=e\left(B /\left(\mathfrak{b}_{0}+x_{1} B\right) .\right.\right.
$$

For the equations (b) and (c) of (i), note that $c_{0}(I)=e\left(B /\left(\mathfrak{b}_{0}+x_{1} B\right)\right)$ by the intersection algorithm, $\mathfrak{b}_{1}=\left(\mathfrak{b}_{0}+x_{1} B\right):\langle I B\rangle=B$ and $e_{1}(\mathfrak{m} \mid I)=0$ by Remark 3. It follows that $c_{0}(I)=e_{0}(I \mid \mathfrak{m})-e_{1}(\mathfrak{m} \mid I)$.

Now let us consider the case $d \geq 2$. Then $\bar{B}:=B /\left(0:\langle I B\rangle+x_{1} B\right)$ is of dimension at most $d-1 \geq 1$. If $\operatorname{dim} \bar{B}<d-1$, then $e_{k}(I B \mid \mathfrak{m} B, B)=0$ for $k=$ $0, \ldots, d-1$ (see Remark 2). If $\operatorname{dim} \bar{B}=d-1$, then, using the genericity of $x_{1}$, by Lemmas 2.2 and 2.3 we have $e_{k}(I B \mid \mathfrak{m} B, B)=e_{k}(I \bar{B} \mid \mathfrak{m} \bar{B}, \bar{B})$ for $k=0, \ldots, d-2$. If $\operatorname{dim} \bar{B}>\operatorname{dim}(\bar{B} / 0:\langle I \bar{B}\rangle)$, then $c_{k}(I \bar{B})=e_{k}(I \bar{B} \mid \mathfrak{m} \bar{B})=e_{k}(\mathfrak{m} \bar{B} \mid I \bar{B})=0$ for $k=0, \ldots, d-2$ (see Remark 2), and if $\operatorname{dim} \bar{B}=\operatorname{dim}(\bar{B} / 0:\langle I \bar{B}\rangle)$, then by Lemmas 2.3, 2.2 and the induction hypothesis it holds

$e_{d-k}(I B \mid \mathfrak{m} B)=e_{d-1-(k-1)}(I \bar{B} \mid \mathfrak{m} \bar{B})=e\left(\bar{B} /\left(\overline{\mathfrak{b}}_{k-2}+x_{k} \bar{B}\right)\right)=e\left(B / \mathfrak{b}_{k-1}+x_{k} B\right)$

for $k-1=1, \ldots, s-1$. This proves the assertion (a) of (i) for $k=2, \ldots, s$.

It remains to prove the assertion (a) of (i) for $k=1$, that is,

$$
e_{d-1}(I B \mid \mathfrak{m} B)=e\left(B /\left(\mathfrak{b}_{0}+x_{1} B\right)\right)=e\left(B /\left(0:\langle I B\rangle+x_{1} B\right)\right)
$$

if $\operatorname{dim} B=\operatorname{dim}\left(B / \mathfrak{b}_{0}\right)=\operatorname{dim}(B /(B / 0:\langle I B\rangle)$ or

$$
e_{d-1}(I B \mid \mathfrak{m} B)=0 \quad \text { if } \operatorname{dim} B>\operatorname{dim}\left(B / \mathfrak{b}_{0}\right) .
$$

The latter is clear by Remark 2. So let us assume that $\operatorname{dim} B=\operatorname{dim}\left(B / \mathfrak{b}_{0}\right)$. Then by Lemma 2.3,

$$
e_{d-1}(I B \mid \mathfrak{m} B)=e_{d-1}(I B \mid \mathfrak{m} B, B / 0:\langle I B\rangle) .
$$

By Lemma 2.2, there are elements $y_{1}, \ldots, y_{d-1} \in \mathfrak{m}$ such that

$$
\begin{aligned}
e_{d-1}(I B \mid \mathfrak{m} B, B / 0:\langle I B\rangle) & =e_{d-2}\left(I B \mid \mathfrak{m} B, B / 0:\langle I B\rangle+y_{1} B\right)=\cdots \\
& =e_{0}\left(I B \mid \mathfrak{m} B, B /\left(0:\langle I B\rangle+\left(y_{1}, \ldots, y_{d-1}\right) B\right)\right)
\end{aligned}
$$


and such that, for $k=1, \ldots, d-1$, all $(d-k)$-dimensional associated prime ideals of $0:\langle I B\rangle+\left(y_{1}, \ldots, y_{k}\right) B$ do not contain $I B$.

In order to see $(9)$, we distinguish three cases for $k$ :

If $k<d-\operatorname{dim}(A / I)$ then

$$
\operatorname{dim}\left(B /\left(0:\langle I B\rangle+\left(y_{1}, \ldots, y_{k}\right) B\right)=d-k>\operatorname{dim}(B / I B)=\operatorname{dim}(A / I),\right.
$$

hence a $(d-k)$-dimensional prime ideal cannot contain $I B$.

If $k=d-\operatorname{dim}(A / I)$ and $y_{k}$ has been chosen outside all highest-dimensional associated prime ideals of $I B$, then a prime ideal which contains both $y_{k}$ and $I B$ has dimension strictly smaller than $\operatorname{dim}(A / I)$, thus, taking into account that

$$
\operatorname{dim}\left(B /\left(0:\langle I B\rangle+\left(y_{1}, \ldots, y_{k}\right) B\right)\right)=\operatorname{dim}(A / I)
$$

there is no highest-dimensional prime ideal of $0:\langle I B\rangle+\left(y_{1}, \ldots, y_{k}\right) B$ that contains $I B$.

Finally, if $d-\operatorname{dim}(A / I)<k \leq d-1$ and $y_{k}$ has been chosen outside all highest-dimensional associated prime ideals of the $(d-k)$-dimensional ideal $I B+\left(y_{d-\operatorname{dim}(A / I)}, \ldots, y_{k-1}\right) B$, then a prime ideal which contains both $y_{k}$ and $I B+\left(y_{d-\operatorname{dim}(A / I)}, \ldots, y_{k-1}\right) B$ has dimension strictly smaller than $d-k$. Hence, as above, there is no highest-dimensional prime ideal of $0:\langle I B\rangle+\left(y_{1}, \ldots, y_{k}\right) B$ that contains $I B$ which proves (9).

By Lemma 2.3,

$$
\begin{gathered}
e_{0}\left(I B \mid \mathfrak{m} B, B /\left(0:\langle I B\rangle+\left(y_{1}, \ldots, y_{d-1}\right) B\right)\right) \\
=e_{0}\left(I B \mid \mathfrak{m} B, B /\left(0:\langle I B\rangle+\left(y_{1}, \ldots, y_{d-1}\right) B\right):\langle\mathfrak{m} B\rangle\right),
\end{gathered}
$$

and the image of $I B$ in the one-dimensional Cohen-Macaulay ring

$$
B /\left(0:\langle I B\rangle+\left(y_{1}, \ldots, y_{d-1}\right) B:\langle\mathfrak{m} B\rangle\right)
$$

is primary to the maximal ideal, hence

$$
\begin{aligned}
& e_{0}\left(I B \mid \mathfrak{m} B, B /\left(0:\langle I B\rangle+\left(y_{1}, \ldots, y_{d-1}\right) B\right):\langle\mathfrak{m} B\rangle\right) \\
= & e\left(I B, B /\left(0:\langle I B\rangle+\left(y_{1}, \ldots, y_{d-1}\right) B\right):\langle\mathfrak{m} B\rangle\right) \\
& \quad(\text { by }[26, \text { Lemma 2.2] or }[36, \text { Lemma 3.1]) } \\
= & e\left(x_{1} B, B /\left(0:\langle I B\rangle+\left(y_{1}, \ldots, y_{d-1}\right) B:\langle\mathfrak{m} B\rangle\right)\right) \\
& \quad\left(\text { since } x_{1} \text { is a generic element of } I B\right) \\
= & \operatorname{length}\left(B /\left(0:\langle I B\rangle+\left(y_{1}, \ldots, y_{d-1}\right) B:\langle\mathfrak{m} B\rangle\right)+x_{1} B\right) \\
& \quad(\text { by the Cohen-Macaulay property) } \\
= & e\left(\left(x_{1}, y_{1}, \ldots, y_{d-1}\right), B /(0:\langle I B)) \quad(\text { by }[7, \text { Theorem] })\right. \\
= & e_{1}\left(\mathfrak{m} \mid I, B /(0:\langle I B))=e\left(B /\left(0:\langle I B\rangle+x_{1} B\right)\right) \quad(\text { by }[36, \text { Proposition } 4.1]) .\right.
\end{aligned}
$$

Together with (8), (9) and (2) this proves that

$$
e_{d-1}(I B \mid \mathfrak{m} B)=e\left(B /\left(0:\langle I B\rangle+x_{1} B\right)\right)=e\left(B /\left(\mathfrak{b}_{0}+x_{1} B\right)\right) .
$$


The statements of (i) concerning $e_{k-1}(\mathfrak{m} \mid I, A)$ (are essentially [36, Theorem 3.4 and our Remark 2] or can be proved as the statements of (i) concerning $e_{d-k}(I \mid \mathfrak{m}, A)$ by induction using Lemma 2.3, Lemma 2.2 and Remark 2.

For (ii) it is sufficient to remark that $B /\left(\mathfrak{b}_{s-1}+x_{s} B\right):\langle I B\rangle$ is the zero ring.

Remark 4. Theorem 2.4 expresses the local degrees of all varieties appearing in the construction of the Segre cycles of [15] as mixed multiplicities. Thus no general hypersurfaces are needed. In fact, if we use notation of [15] and set $A=\mathcal{O}_{Y, 0}$, then, by applying Theorem 2.4 to the cycles in [15, Lemma 2.2], we have a complete description of the local degrees of the cycles by mixed multiplicities:

$$
\begin{aligned}
\operatorname{mult}_{0}\left(\Lambda_{k}^{\mathbf{g}}(I, Y)\right) & =c_{d-k}(I, A), \quad(\text { the } k \text { th Segre number of }(I, Y)), \\
\operatorname{mult}_{0}\left(V\left(\left.g_{k}\right|_{P_{k-1}^{\mathrm{g}}(I, Y)}\right)\right) & =e_{d-k}(I \mid \mathfrak{m}, A), \\
\operatorname{mult}_{0}\left(P_{k}^{\mathbf{g}}(I, Y)\right) & =e_{k}(\mathfrak{m} \mid I, A), \quad(\text { the } k \text { th polar multiplicity of }(I, Y)) .
\end{aligned}
$$

Then, by taking degrees in the equality of cycles from [15, Lemma 2.2]

$$
\begin{aligned}
\Lambda_{k}^{\mathbf{g}}(I, Y) & =\left[V\left(\left.g_{k}\right|_{\left.P_{k-1}^{\mathbf{g}}(I, Y)\right)}\right]-\left[P_{k}^{\mathbf{g}}(I, Y)\right], \quad k=0, \ldots, d-1,\right. \\
\Lambda_{d}^{\mathbf{g}}(I, Y) & =\left[V\left(\left.g_{d}\right|_{P_{d-1}^{\mathbf{g}}(I, Y)}\right)\right]
\end{aligned}
$$

we obtain the formula given in Theorem 2.4, (i) (c)

$$
c_{d-k}(I, A)=e_{d-k}(I \mid \mathfrak{m}, A)-e_{k}(\mathfrak{m} \mid I, A) .
$$

Theorem 2.4 implies that the new mixed multiplicities $e_{k}(I \mid \mathfrak{m})$ are preserved by passing to an arbitrary reduction of the ideal $I$.

Corollary 2.5. Let $J$ be an arbitrary reduction of $I$. Then

$$
e_{k}(I \mid \mathfrak{m})=e_{k}(J \mid \mathfrak{m}), \quad e_{k}(\mathfrak{m} \mid I)=e_{k}(\mathfrak{m} \mid J), \quad c_{k}(I)=c_{k}(J)
$$

for all $k=0, \ldots, d-1$.

Proof. The second equalities are a special case of [36, Corollary 3.8], the third ones are [9, Proposition 2.7], see also [37, Corollary 11.5], and $e_{k}(I \mid \mathfrak{m})=e_{k}(J \mid \mathfrak{m})$ follows immediately from Theorem 2.4.

Corollary 2.6. Let $d=\operatorname{dim} A$ and $s=s(I)$ be the analytic spread of $I$. Then $e_{k}(I \mid \mathfrak{m})=0$ for $k=0, \ldots, d-s-1$ and $e_{d-s}(I \mid \mathfrak{m})=c_{d-s}(I)$.

Furthermore, if $A$ is quasi-unmixed (or formally equidimensional), that is, equidimensional and universally catenary, then

$$
\begin{gathered}
e_{k}(I \mid \mathfrak{m})>0 \text { for } k=d-s, \ldots, d-1 \text { and } \\
e_{\operatorname{dim}(A / I)}(I \mid \mathfrak{m}) \geq \cdots \geq e_{d-2}(I \mid \mathfrak{m}) \geq e_{d-1}(I \mid \mathfrak{m})>0 .
\end{gathered}
$$


Proof. The corollary is immediate from Theorem 2.4, (i)(a),(c) and (ii). For the last assertion, observe that by $[22,(18.17)], A$ is quasi-unmixed if and only if it is equidimensional and universally catenary, and if this is the case, then for each proper ideal $I \subset A$ the associated graded ring $G=G_{I}(A)$ is also quasi-unmixed, see $[22,(18.24)]$, which implies that for all graded prime ideals $P \subset G$ it holds $\operatorname{dim} G / P+\operatorname{dim} G_{P}=\operatorname{dim} G$, hence $c_{d-s}(I)=e_{d-s}(I \mid \mathfrak{m})>0$, see [3, Proposition 2.3(ii)], and the positivity of the other mixed multiplicities follows by Theorem 2.4, (i)(a) and their monotony by Theorem 2.4,(iii) and [13, Theorem 1.2.11].

In a discussion with $\mathrm{C}$. Bivià-Ausina the first two authors realized that the new mixed multiplicity $e_{k}(I \mid \mathfrak{m}, A)$, where $I$ is not necessarily $\mathfrak{m}$-primary, equals the $j$-multiplicity, that is $c_{0}$, of the extension of $I$ in the quotient ring of $A$ by $k$ general elements of $\mathfrak{m}$. This result can be seen as an algebraic generalization of Teissier's $\mu^{*}$-sequence [35, Définition 1.5, p. 300, Prop. 2.10, p. 315] of an isolated hypersurface singularity.

Corollary 2.7. Let $d:=\operatorname{dim}(A)>1$ and $\left(y_{1}, \ldots, y_{n}\right), n \geq d$, be a transformed set of generators for $\mathfrak{m} B$, that is, $y_{1}, \ldots, y_{n}$ are general elements which generate $\mathfrak{m} B$, see the definition before (3). Then

$$
e_{k}(I \mid \mathfrak{m}, A)=e_{k}(I B \mid \mathfrak{m} B, B)=c_{0}\left(I B, B /\left(y_{1}, \ldots, y_{k}\right) B\right) \quad \text { for } k=0, \ldots, d-1 .
$$

Furthermore, if $A$ is Cohen-Macaulay and $\left(x_{1}, \ldots, x_{n}\right)$ is a transformed set of generators for $I B$, then

$$
\begin{aligned}
e_{k}(I \mid \mathfrak{m}, A) & =e\left(\left(x_{1}, \ldots, x_{d-k}, y_{1}, \ldots, y_{k}\right) B\right) \quad \text { for } k=\operatorname{dim}(A / I), \ldots, d-1, \\
& =\sigma(\underbrace{I B, \ldots, I B}_{d-k \text { times }}, \underbrace{\mathfrak{m} B, \ldots, \mathfrak{m} B}_{k \text { times }}) \\
& \text { (Rees' mixed multiplicity of Bivià-Ausina [6, Definition 2.4]), }
\end{aligned}
$$

where $\left(y_{1}, \ldots, y_{k}\right)$ is defined to be $(0)$ if $k=0$.

Proof. We prove the first assertion of the corollary by induction on $d$. The case $d=1$ is clear by Theorem 2.4(i)(a) (or (iii) if $s(I)<d$ ) which states $e_{0}(I \mid \mathfrak{m}, A)=c_{0}(I, A)=c_{0}(I B, B)$ without any restriction on $d$.

Now assume that $d>1, k>0$, and that the first assertion of the corollary is true for $d-1$. By Lemma 2.2 we have $e_{k}(I B \mid \mathfrak{m}, B)=e_{k-1}\left(I B \mid \mathfrak{m} B, B / y_{k} B\right)$. Putting this together with the induction hypothesis $e_{k-1}\left(I B \mid \mathfrak{m}, B / y_{k} B\right)=$ $c_{0}\left(I B, B /\left(y_{1}, \ldots, y_{k-1}, y_{k}\right) B\right)$ gives the result.

The second assertion for $k=0$, that is, $\operatorname{dim}(A / I)=0$, states that

$$
e_{0}(I \mid \mathfrak{m}, A)=e\left(\left(x_{1}, \ldots, x_{d}\right) B\right) .
$$

By a classical result of Northcott and Rees [31] the latter multiplicity is equal to $e(I B)$. In fact, $\operatorname{dim}(A / I)=0$ implies that $I$ is $\mathfrak{m}$-primary and that $\left(x_{1}, \ldots, x_{d}\right) B$ is a minimal reduction of $I B$. By Rees [32, Lemma 2.4] it is known that $e_{0}(I \mid \mathfrak{m}, A)=e(I)=e(I B)$. 
In order to prove the second assertion in the case $k>0$, we note that $d-\operatorname{dim}(A / I) \leq s$, hence by Theorem $2.4(\mathrm{i})(\mathrm{a})$

$$
e_{d-k}(I \mid \mathfrak{m}, A)=e\left(B /\left(\mathfrak{b}_{k-1}+x_{k} B\right)\right) \quad \text { for } k=1, \ldots, d-\operatorname{dim}(A / I),
$$

and the Cohen-Macaulay property of $A$ (and hence of $B$ ) implies that $\mathfrak{b}_{k-1}=$ $\left(x_{1}, \ldots, x_{k-1}\right) B$ for $k=1, \ldots, d-\operatorname{dim}(A / I)$ and that

$$
e_{d-k}(I \mid \mathfrak{m}, A)=e\left(B /\left(x_{1}, \ldots, x_{k}\right) B\right)=e\left(\left(x_{1}, \ldots, x_{k}, y_{1}, \ldots, y_{d-k}\right) B\right)
$$

for $k=1, \ldots, d-\operatorname{dim}(A / I)$.

In the special case when the ideal $I$ is graded and generated by forms of the same degree we obtain the following two corollaries of Theorem 2.4.

Corollary 2.8. Let $A$ be a standard graded algebra such that $\left(A_{0}, \mathfrak{n}\right)$ is an Artinian local ring and $\mathfrak{m}=\left(\mathfrak{n}, A_{1}\right)$ is the unique homogeneous maximal ideal of $A$ and $I \subset A$ a proper ideal generated by forms of the same degree $r$ and which is not contained in any highest dimensional prime ideal of $A$. Then

$$
e_{k}(\mathfrak{m} \mid I, A)=r^{k} e(A)
$$

for $0 \leq k<\operatorname{dim}(A)-\operatorname{dim}(A / I)$.

Proof. We use the notation of Theorem 2.4. Since $I$ is not contained in any highest dimensional prime ideal of $A$, by Theorem 2.4 (i) we have

$$
e_{0}(\mathfrak{m} \mid I, A)=e\left(B / \mathfrak{b}_{0}\right)=e(B)=e(A)
$$

and for each $k=1, \ldots, \operatorname{dim} A-\operatorname{dim}(A / I)-1$, by the definition of $\mathfrak{b}_{k}$ (see $\left.(3)\right)$, we have $\operatorname{dim}\left(B / \mathfrak{b}_{k}\right)=d-k$ and

$$
e_{k}(\mathfrak{m} \mid I, A)=e\left(B / \mathfrak{b}_{k}\right)=r \cdot e\left(B / \mathfrak{b}_{k-1}\right) .
$$

Corollary 2.9. Let $A$ be a standard graded algebra such that $\left(A_{0}, \mathfrak{n}\right)$ is an Artinian local ring and $\mathfrak{m}=\left(\mathfrak{n}, A_{1}\right)$ is the unique homogeneous maximal ideal of $A$ and $I \subset A$ be a proper ideal of analytic spread $s=s(I)$ generated by forms of the same degree $r$. Let $d=\operatorname{dim}(A) \geq 1$. Then we have

$$
\begin{aligned}
& e_{d-k}(I \mid \mathfrak{m}, A)=r \cdot e_{k-1}(\mathfrak{m} \mid I, A) \quad \text { for } \quad k=1, \ldots, s \quad \text { and } \\
& e_{d-k}(I \mid \mathfrak{m}, A)=e_{k-1}(\mathfrak{m} \mid I, A)=0 \quad \text { for } \quad k=s+1, \ldots, d-1 .
\end{aligned}
$$

Proof. By Theorem 2.4

$$
e_{d-k}(I \mid \mathfrak{m}, A)=e\left(B /\left(\mathfrak{b}_{k-1}+x_{k} B\right)\right)=r \cdot e\left(B / \mathfrak{b}_{k-1}\right)=r \cdot e_{k-1}(\mathfrak{m} \mid I, A) .
$$




\section{Mixed multiplicities, generalized Samuel multiplicities and degree of fiber cones}

In this section we prove that the classical mixed multiplicities $e_{k}(\mathfrak{m} \mid I, A)$ are equal to the generalized Samuel multiplicities of an ideal in the Rees algebra $R_{I}(A)$, see Theorem 3.1. As an application we describe the degree of the fiber cone of an ideal in terms of a classical mixed multiplicity, see Theorem 3.3 and Remark 5. This generalizes a recent result of Jeffries, Montaño and Varbaro [25, Theorem 3.1].

Theorem 3.1. Let $A$ be a d-dimensional Noetherian local ring $(A, \mathfrak{m})$ or a standard graded algebra $A$ such that $\left(A_{0}, \mathfrak{n}\right)$ is an Artinian local ring and $\mathfrak{m}=$ $\left(\mathfrak{n}, A_{1}\right)$ is the unique homogeneous maximal ideal of $A$.

Let $I \subset A$ be a proper ideal and let $R=R_{I}(A)$ the Rees algebra of $A$ with respect to $I$ and let $\mathfrak{M}=(\mathfrak{m}, I t) R$ the unique homogeneous maximal ideal of $R$.

Then for each $i, j \geq 0$ there is an isomorphism

$$
G_{\mathfrak{M}}^{i}\left(G_{\mathfrak{m} R}^{j}(R)\right) \cong G_{\mathfrak{m} R}^{j}\left(R_{i}\right)
$$

Moreover if $\operatorname{dim} R=d+1$, then

$$
c_{k+1}(\mathfrak{m} R, R)=e_{k}(\mathfrak{m} \mid I, A), \quad k=0, \ldots, d-1 .
$$

Proof. We consider

$$
G_{\mathfrak{M}}^{i}\left(G_{\mathfrak{m} R}^{j}(R)\right) \cong \frac{\mathfrak{M}^{i} \mathfrak{m}^{j} R+\mathfrak{m}^{j+1} R}{\mathfrak{M}^{i+1} \mathfrak{m}^{j} R+\mathfrak{m}^{j+1} R}
$$

and we observe that

$$
\begin{gathered}
\mathfrak{M}^{i}=\left(\mathfrak{m} R, R_{1}\right)^{i}=\mathfrak{m}^{i} R_{0} \oplus \mathfrak{m}^{i-1} R_{1} \oplus \cdots \oplus \mathfrak{m} R_{i-1} \oplus\left(\oplus_{k \geq i} R_{k}\right), \\
\mathfrak{M}^{i} \mathfrak{m}^{j} R=\mathfrak{m}^{i+j} R_{0} \oplus \mathfrak{m}^{i+j-1} R_{1} \oplus \cdots \oplus \mathfrak{m}^{j+1} R_{i-1} \oplus\left(\oplus_{k \geq i} \mathfrak{m}^{j} R_{k}\right), \\
\mathfrak{M}^{i+1} \mathfrak{m}^{j} R=\mathfrak{m}^{i+j+1} R_{0} \oplus \mathfrak{m}^{i+j} R_{1} \oplus \cdots \oplus \mathfrak{m}^{j+1} R_{i} \oplus\left(\oplus_{k \geq i+1} \mathfrak{m}^{j} R_{k}\right), \\
\mathfrak{M}^{i} \mathfrak{m}^{j} R+\mathfrak{m}^{j+1} R=\mathfrak{m}^{j} R_{i} \oplus\left(\oplus_{k \geq i+1} \mathfrak{m}^{j} R_{k}\right) \oplus\left(\oplus_{k=0}^{i-1} \mathfrak{m}^{j+1} R_{k}\right), \\
\mathfrak{M}^{i+1} \mathfrak{m}^{j} R+\mathfrak{m}^{j+1} R=\mathfrak{m}^{j+1} R_{i} \oplus\left(\oplus_{k \geq i+1} \mathfrak{m}^{j} R_{k}\right) \oplus\left(\oplus_{k=0}^{i-1} \mathfrak{m}^{j+1} R_{k}\right),
\end{gathered}
$$

hence

$$
G_{\mathfrak{M}}^{i}\left(G_{\mathfrak{m} R}^{j}(R)\right) \cong \mathfrak{m}^{j} R_{i} / \mathfrak{m}^{j+1} R_{i}=G_{\mathfrak{m} R}^{j}\left(R_{i}\right) .
$$

The last assertion follows immediately from the above isomorphism and the definition of these numbers.

Corollary 3.2. Let $A$ be a d-dimensional Noetherian local ring $(A, \mathfrak{m})$ or a standard graded algebra $A$ such that $\left(A_{0}, \mathfrak{n}\right)$ is an Artinian local ring and $\mathfrak{m}=$ $\left(\mathfrak{n}, A_{1}\right)$ is the unique homogeneous maximal ideal of $A$.

Let $I \subset A$ be a proper ideal of analytic spread $s=s(I)$ and let $R=R_{I}(A)$ the Rees algebra of $A$ with respect to $I$ and let $\mathfrak{M}=(\mathfrak{m}, I t) R$ the unique homogeneous maximal ideal of $R$. 
(i) If $\operatorname{dim} R=\operatorname{dim} A+1$, then

$$
e_{s-1}(\mathfrak{m} \mid I, A)=\sum_{\mathfrak{P}} e(\mathfrak{M}, R / \mathfrak{P}) \cdot e\left(\mathfrak{m} R_{\mathfrak{P}}, R_{\mathfrak{P}}\right),
$$

where $\mathfrak{P}$ runs through all highest dimensional associated prime ideals of $R / \mathfrak{m} R$ such that $\operatorname{dim} R / \mathfrak{P}+\operatorname{dim} R_{\mathfrak{P}}=\operatorname{dim} R$ and

$$
e_{s}(\mathfrak{m} \mid I, A)=\cdots=e_{d-1}(\mathfrak{m} \mid I, A)=0 .
$$

(ii) If $\operatorname{dim} A / I<d$, then

$$
e_{0}(\mathfrak{m} \mid I, A)=e(A) .
$$

Proof. The Corollary follows from the above Theorem and from [3, Proposition 2.3]. The assertion (ii) has been proved by Katz and Verma [26, Lemma 2.2] under the assumption $\mathrm{ht}(I)>0$, but their proof holds also under the weaker assumption $\operatorname{dim} A / I<d$.

Theorem 3.3. Let $A$ be a standard graded domain over a field $k$ with unique homogeneous maximal ideal $\mathfrak{m}$. Let $I \subset A$ be a proper ideal of analytic spread $s=s(I)$ generated by forms of the same degree $r$ and let $R=R_{I}(A)$ the Rees algebra of $A$ with respect to $I$.

Then

$$
e_{s-1}(\mathfrak{m} \mid I, A)=e(R / \mathfrak{m} R) \cdot e\left(R_{\mathfrak{m} R}\right) .
$$

Proof. Being $A$ a domain, the Rees algebra $R$ is a domain too, since it is a subring of the integral domain $A[t]$. The quotient $\operatorname{ring} R / \mathfrak{m} R$ is isomorphic to $k\left[f_{1}, \cdots, f_{s}\right] \subset k\left[A_{r}\right]$, hence it is a domain, that is, $\mathfrak{m} R$ is a prime ideal in the integral domain $R$. It follows that

$$
\operatorname{dim} R / \mathfrak{m} R+\operatorname{dim} R_{\mathfrak{m} R}=\operatorname{dim} R
$$

(see, for example, [29, $14 \mathrm{H}]$ ), hence by Corollary 3.2,

$$
e_{s-1}(\mathfrak{m} \mid I, A)=e(\mathfrak{M}, R / \mathfrak{m} R) \cdot e\left(\mathfrak{m} R_{\mathfrak{m} R}, R_{\mathfrak{m} R}\right)=e(R / \mathfrak{m} R) \cdot e\left(R_{\mathfrak{m} R}\right) .
$$

Remark 5. (Fiber cone) Let $A$ be a Noetherian local ring $(A, \mathfrak{m})$ or a standard graded algebra $A$ such that $\left(A_{0}, \mathfrak{n}\right)$ is a local ring and $\mathfrak{m}=\left(\mathfrak{n}, A_{1}\right)$ is the homogeneous maximal ideal of $A$. Given an ideal $I \subset A$ (homogeneous if $A$ is graded), the fiber cone or special fiber ring of $I$ is the graded algebra

$$
F(I)=\bigoplus_{i \geq 0} I^{i} / \mathfrak{m} I^{i}=R_{I}(A) / \mathfrak{m} R_{I}(A)=G_{I}(A) / \mathfrak{m} G_{I}(A)
$$

of Krull-dimension $s$.

Theorem 3.3 says that the degree (or multiplicity) of the fiber cone $F(I)$ is

$$
e(F(I))=e_{s-1}(\mathfrak{m} \mid I, A) / e\left(R_{\mathfrak{m} R}\right) .
$$


Corollary 3.4. Under the assumption of Theorem 3.3 one has

$$
e(F(I)) \leq e_{s-1}(\mathfrak{m} \mid I, A)
$$

and equality holds if and only if $R_{\mathfrak{m} R}$ is a regular local ring.

Proof. It is an immediate consequence of Theorem 3.3, since $e\left(R_{\mathfrak{m} R}\right) \geq 1$ and equality holds if and only if $R_{\mathfrak{m} R}$ is a regular local ring, since $R_{\mathfrak{m} R}$ is an integral domain (see [30, Theorem 40.6]).

As a consequence of Theorem 3.3 and Corollary 2.9 we obtain a generalization of [25, Theorem 3.1] to ideals of not necessarily maximal analytic spread.

Corollary 3.5. Under the assumption of Theorem 3.3, let us assume that the ideal I has maximal analytic spread $d$. Then

$$
c_{0}(I, A)=r \cdot e_{d-1}(\mathfrak{m} \mid I, A)=r \cdot e(F(I)) \cdot e\left(R_{\mathfrak{m} R}\right) .
$$

Proof. Under the assumption $s(I)=d$, we have

$$
\begin{aligned}
c_{0}(I, A) & =e_{0}(I \mid \mathfrak{m}, A) & (\text { Theorem 2.4 (i) (a)) } \\
& =r \cdot e_{d-1}(\mathfrak{m} \mid I, A) & (\text { Corollary 2.9) } \\
& =r \cdot e(F(I)) e\left(R_{\mathfrak{m} R}\right) & (\text { Theorem 3.3) }
\end{aligned}
$$

\section{Segre classes}

Let $K$ be an algebraically closed field, $\mathbb{P}^{n}$ the projective $n$-space over $K, Y$ a $(d-1)$-dimensional closed subvariety of $\mathbb{P}^{n}$ and $X \nsubseteq Y$ a $q$-dimensional closed subscheme. For the definition of the Segre class $s(X, Y)$ of $X$ in $Y$ we refer to [14, Chapter 4]. If $i: X \hookrightarrow \mathbb{P}^{n}$ is the inclusion, then the push-forward of $s(X, Y)$ to $\mathbb{P}^{n}$ is a class in $A_{*}\left(\mathbb{P}^{n}\right)$ and can be written as

$$
\begin{aligned}
i_{*} s(X, Y) & =s_{0}\left[\mathbb{P}^{0}\right]+s_{1}\left[\mathbb{P}^{1}\right]+\cdots+s_{q}\left[\mathbb{P}^{q}\right]= \\
& =s^{d-1}\left[\mathbb{P}^{0}\right]+s^{d-2}\left[\mathbb{P}^{1}\right]+\cdots+s^{d-1-q}\left[\mathbb{P}^{q}\right]+\cdots+s^{0}\left[\mathbb{P}^{d-1}\right] .
\end{aligned}
$$

Note that $s_{k}=s^{d-1-k}(k=0, \ldots, d-1)$, that is, the upper index refers to the codimension in $Y$. Obviously $s^{d-2-q}=\cdots=s^{0}=0$.

Furthermore, let $I(X)=\left(f_{1}, \ldots, f_{t}\right) \subset K\left[x_{0}, \ldots, x_{n}\right]$ be the defining ideal of $X, r_{i}=\operatorname{deg} f_{i}$ for $i=1, \ldots, t, A=K\left[x_{0}, \ldots, x_{n}\right] / I(Y)$, and $\mathfrak{m}=\left(x_{0}, \ldots, x_{n}\right) A$. Then (Krull-) $\operatorname{dim} A=d$. The scheme $X$ can be defined by an ideal $I$ generated by forms of the same degree $r, r \geq \max \left(r_{1}, \ldots, r_{t}\right)$, for example by replacing each form $f_{i}$ with $r_{i}<r$ by the $n+1$ forms $\tilde{f}_{i, j}=x_{j}{ }^{r-r_{i}} f_{i}, j=0, \ldots, n$. We denote the forms of degree $r$ which generate $I$ simply by $\tilde{f}_{0}, \ldots, \tilde{f}_{m}$. Let $\phi$ be the rational map $Y \rightarrow \mathbb{P}^{m}$ defined by

$$
p \mapsto\left[\tilde{f}_{0}(p): \cdots: \tilde{f}_{m}(p)\right] .
$$


According to Harris [18, Example 19.4] one can associate to $\phi$ projective degrees $\operatorname{deg}_{0}(\phi), \ldots, \operatorname{deg}_{d-1}(\phi)$, while Aluffi $[4,3.2]$ introduces the so-called shadow of (the closure of) the graph $\Gamma_{\phi}$ of $\phi$, that is, the push-forward of $\left[\Gamma_{\phi}\right]$ by the projection map onto the first factor of $Y \times \mathbb{P}^{m}$ to a class

$$
[G]=g_{0}+g_{1} h+\cdots+g_{d-1} h^{d-1} \in A_{*}(Y) .
$$

Then it holds the following proposition.

Proposition 4.1. With the preceding notation and denoting by $s$ the analytic spread of $I$ and by $\mathfrak{b}_{k} \subseteq B$ the ideals of the residual sets $V\left(\mathfrak{b}_{k}\right)$ in the intersection algorithm (3), one has

$$
\operatorname{deg}_{d-1-k}(\phi)=g_{k}=e_{k}(\mathfrak{m} \mid I, A)=e\left(\mathfrak{b}_{k}\right)=\operatorname{deg} V\left(\mathfrak{b}_{k}\right) \quad \text { for } k=0, \ldots, s-1
$$

and

$$
\operatorname{deg}_{d-1-k}(\phi)=g_{k}=e_{k}(\mathfrak{m} \mid I, A)=0 \quad \text { for } k=s, \ldots, d-1 .
$$

Proof. By Helmer [20, Section 3.2, (10)] and by Theorem 2.4 the proposition follows.

The degrees of the Segre classes of subschemes of arbitrary projective varieties can be computed by computing generalized Samuel multiplicities or mixed multiplicities of ideals, that is, by computing bivariate Hilbert polynomials. Vice versa generalized Samuel multiplicities and the mixed multiplicities can be calculated by the Segre classes, that is, all these "characteristic numbers" carry the same information.

In the following theorem we collect the conversion formulas (11)-(14) for the several characteristic numbers. As for these formulas, it seems that commutative algebraists and algebraic geometers have ignored each other.

Formulas (11) and (12) are more or less known since the late 1980's, when van Gastel [16] discovered the relationship between between the intersection theory of Fulton and MacPherson ([14]), which relies on the notion of Segre classes, and that of Stückrad and Vogel ([34], [41], [13]), which is based on an intersection algorithm. In 1997, Achilles and Manaresi [3] expressed the degrees of the Stückrad-Vogel cycle by the leading coefficients of a bivariate Hilbert polynomial, the so-called generalized Samuel multiplicities. This allowed a computation of the degrees of the Segre class (see [3, Corollary 4.3] and Flenner, O'Carroll, Vogel [13, Corollary 2.4.7]) and was implemented in the first version of the REDUCE script Segre [1].

In 2003, Aluffi [4, Proposition 3.1] used the relation (13) for computing Segre classes. Also Helmer [19, p. 18], [20, p. 11, (17)] (who quoted Vogel [41] in [19]), presented (13) in the case $Y=\mathbb{P}^{n}$. The reverse formula (14) can be found in Eklund, Jost and Peterson [11, Theorem 3.2] (in the case $Y=\mathbb{P}^{n}$ ) and Harris [17, Proposition 4]. It seems that all these authors are not aware of van Gastel's paper [16].

We shall show that, once one has van Gastel's result [16, Corollary 3.7], all the formulas of the following theorem are an immediate consequence of the 
Stückrad-Vogel algorithm (Theorem 2.4). In the formulas, in order to include $c_{0}(I, A)$, an $s_{-1}$ will appear. If the prime ideal of $X$ is generated by forms of the same degree $r$, then $s_{-1}$ can be interpreted as $s_{0}$ of the projective cone of $X$ in the projective cone of $Y \subset \mathbb{P}^{n+1}$.

Theorem 4.2. For $k=0, \ldots, d$, maintaining the previous notation, setting $c_{k}=c_{k}(I, A)$ and using the convention that for $m, n \in \mathbb{Z},\left(\begin{array}{c}m \\ n\end{array}\right)=0$ if $-1 \leq m<$ $n,\left(\begin{array}{c}m \\ -1\end{array}\right)=0$ if $m \geq 0$ and $\left(\begin{array}{c}-1 \\ -1\end{array}\right)=1$, it holds:

$$
\begin{aligned}
s^{k} & =s_{d-1-k}=\sum_{i=0}^{k}\left(\begin{array}{l}
k-1 \\
i-1
\end{array}\right)(-r)^{k-i} c_{d-i}, \\
c_{k} & =\sum_{i=k-1}^{q}\left(\begin{array}{c}
d-k-1 \\
d-i-2
\end{array}\right) r^{i+1-k} s_{i}, \quad c_{d-k}=\sum_{i=0}^{k}\left(\begin{array}{l}
k-1 \\
i-1
\end{array}\right) r^{k-i} s^{i}, \\
s^{k} & =s_{d-1-k}=\sum_{i=1}^{k}\left(\begin{array}{c}
k-1 \\
i-1
\end{array}\right)(-r)^{k-i}\left(e_{d-i}(I \mid \mathfrak{m})-e_{i}(\mathfrak{m} \mid I)\right)= \\
& =\sum_{i=1}^{k}\left(\begin{array}{c}
k-1 \\
i-1
\end{array}\right)(-r)^{k-i}\left(r \cdot e_{i-1}(\mathfrak{m} \mid I)-e_{i}(\mathfrak{m} \mid I)\right), \\
e_{k}(\mathfrak{m} \mid I) & =e(A) r^{k}-\sum_{i=1}^{k}\left(\begin{array}{c}
k \\
i
\end{array}\right) r^{k-i} s^{i}= \\
& =\sum_{i=1}^{d}\left(\begin{array}{l}
d \\
i
\end{array}\right) r^{k-i} s^{i}-\sum_{i=1}^{k}\left(\begin{array}{l}
k \\
i
\end{array}\right) r^{k-i} s^{i}=\sum_{i=1}^{d}\left[\left(\begin{array}{l}
d \\
i
\end{array}\right)-\left(\begin{array}{c}
k \\
i
\end{array}\right)\right] r^{k-i} s^{i} .
\end{aligned}
$$

Proof. The formulas (11) and (12) have been proved by van Gastel [16, Corollary 3.7], see also [13, Corollary 2.4.7].

Formula (13) is easily obtained from (11), since $c_{d}=0$ (by assumption $Y$ is a variety and $X \mp Y$ ), by Theorem $2.4(\mathrm{i})$ (c) and (ii) for all $i=1, \ldots, d$ it holds

$$
c_{d-i}=e_{d-i}(I \mid \mathfrak{m})-e_{i}(\mathfrak{m} \mid I)
$$

and, by Corollary 2.9, for $i=1, \ldots, d$ it holds

$$
e_{d-i}(I \mid \mathfrak{m})=r \cdot e_{i-1}(\mathfrak{m} \mid I) .
$$

Formula (14) is simply the reverse formula of (13) and can be proved by induction on $k$. The case $k=0$, that is $e_{0}(\mathfrak{m} \mid I)=e(A)$, has been proved by Katz and Verma [26, Lemma 2.2]. Now suppose that $k>0$ and

$$
e_{k-1}(\mathfrak{m} \mid I)=e(A) r^{k-1}-\sum_{i=1}^{k-1}\left(\begin{array}{c}
k-1 \\
i
\end{array}\right) r^{k-1-i} s^{i}
$$


Then by the second formula of (12) and (15), (16) it follows that

$$
e_{k}(\mathfrak{m} \mid I)=r \cdot e_{k-1}(\mathfrak{m} \mid I)-\sum_{i=0}^{k}\left(\begin{array}{c}
k-1 \\
i-1
\end{array}\right) r^{k-i} s^{i}
$$

Substituting $e_{k-1}(\mathfrak{m} \mid I)$ by the induction hypothesis (17) gives

$$
\begin{aligned}
e_{k}(\mathfrak{m} \mid I) & =r\left(e(A) r^{k-1}-\sum_{i=1}^{k-1}\left(\begin{array}{c}
k-1 \\
i
\end{array}\right) r^{k-1-i} s^{i}\right)-\sum_{i=0}^{k}\left(\begin{array}{c}
k-1 \\
i-1
\end{array}\right) r^{k-i} s^{i} \\
& =e(A) r^{k}-\sum_{i=1}^{k-1}\left(\left(\begin{array}{c}
k-1 \\
i
\end{array}\right)+\left(\begin{array}{c}
k-1 \\
i-1
\end{array}\right)\right) r^{k-i} s^{i}-s^{k} \\
& =e(A) r^{k}-\sum_{i=1}^{k}\left(\begin{array}{c}
k \\
i
\end{array}\right) r^{k-i} s^{i}
\end{aligned}
$$

which proves the first equality of (14). In particular it holds

$$
0=e_{d}(\mathfrak{m} \mid I)=e(A) r^{d}-\sum_{i=1}^{d}\left(\begin{array}{l}
d \\
i
\end{array}\right) r^{d-i} s^{i}
$$

that is

$$
e(A)=r^{-d} \sum_{i=1}^{d}\left(\begin{array}{l}
d \\
i
\end{array}\right) r^{d-i} s^{i}=\sum_{i=1}^{d}\left(\begin{array}{c}
d \\
i
\end{array}\right) r^{-i} s^{i} .
$$

Substituting $e(A)$ in the first equality of (14) by the preceding expression gives the other equalities of (14).

As an application of Theorem 4.2 we want to compute the degrees of the Segre classes and the Stückrad-Vogel cycles of rational normal scrolls from their mixed multiplicities, which are known by Hoang and Lam [23] for balanced rational normal scrolls. At first we extend their result to arbitrary rational normal scrolls. We will use the following notation:

Rational normal scrolls. Given positive integers $a_{1} \leq \cdots \leq a_{d}$, set

$$
n=\sum_{i=1}^{d}\left(a_{i}+1\right)-1 \quad \text { and } \quad c=n-d=\sum_{i=1}^{d} a_{i}-1 .
$$

Then the associated $d$-dimensional rational normal scroll $S\left(a_{1}, \ldots, a_{d}\right) \subset \mathbb{P}^{n}$ is defined by the ideal $I=I\left(a_{1}, \ldots, a_{d}\right) \subset A=K\left[x_{0}, \ldots, x_{n}\right]$ generated by the 2 -minors of the $2 \times(c+1)$ matrix

$\left(\begin{array}{ccc|ccc|c|ccc}x_{0} & \ldots & x_{a_{1}-1} & x_{a_{1}+1} & \ldots & x_{a_{1}+a_{2}} & \ldots & x_{n-a_{d}} & \ldots & x_{n-1} \\ x_{1} & \ldots & x_{a_{1}} & x_{a_{1}+2} & \ldots & x_{a_{1}+a_{2}+1} & \ldots & x_{n-a_{d}+1} & \ldots & x_{n}\end{array}\right)$,

which has $d$ catalecticant blocks of size $2 \times a_{i}$.

Now we are ready to prove the following theorem, which extends the result of [23, Theorem 2.3] to arbitrary rational normal scrolls, balanced or not. 
Theorem 4.3. Let $S\left(a_{1}, \ldots, a_{d}\right) \subset \mathbb{P}^{n}$ be a d-dimensional rational normal scroll of codimension $c\left(=\sum_{i=1}^{d} a_{i}-1\right)$, let $I \subset A=K\left[x_{0}, \ldots, x_{n}\right]$ be its defining ideal and $\mathfrak{m}=\left(x_{0}, \ldots, x_{n}\right) A$. Then, for $k=0, \ldots, n$, the mixed multiplicities are

$$
e_{k}(\mathfrak{m} \mid I, A)=2^{k}-2 \sum_{i=0}^{k-c-1}\left(\begin{array}{c}
k \\
i
\end{array}\right)-(2 c+1-k)\left(\begin{array}{l}
k \\
c
\end{array}\right) .
$$

Proof. The formula (18) has been proved by Hoang and Lam [23, Theorem 2.3] for ideals in $K\left[x_{0}, \ldots, x_{n}\right]$ generated by the 2 -minors of

$$
S=\left(\begin{array}{ccccc}
x_{0} & x_{1} & x_{2} & \ldots & x_{c} \\
x_{d} & x_{1+d} & x_{2+d} & \ldots & x_{c+d}
\end{array}\right),
$$

where $c+1 \geq d$. Note that our $e_{k}(\mathfrak{m} \mid I, A)$ is $e_{n+1-k}(\mathfrak{m} \mid I)$ in [23]. Dividing $d$ by $(c+1)$ with remainder yields non-negative integers $q$ and $r$ such that $r<d$ and $c+1=q \cdot d+r$. Then the columns of matrix $S$ can be rearranged in $d$ catalecticant blocks as follows:

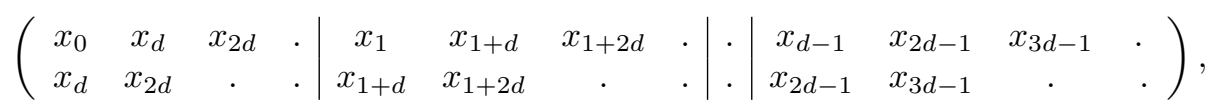

that is, the first $r$ blocks have $q+1$ columns and the last $d-r$ have $q$ columns, see [10, Section 3]. Therefore, according to [18, p. 108], the ideal generated by the 2-minors of the matrix $S$ defines the rational normal scroll $S(q+1, \ldots, q+$ $1, q, \ldots, q)$ of dimension $d$ and codimension $c$. This means that (18) is true for scrolls $S\left(a_{1}, \ldots, a_{d}\right)$ such that $\left|a_{j}-a_{i}\right| \leq 1$ for all $i, j$, the so-called balanced rational normal scrolls.

In order to prove (18) for all rational normal scrolls, we find it convenient to denote the sequence of mixed multiplicities by

$$
e_{*}(\mathfrak{m} \mid I):=e_{*}(\mathfrak{m} \mid I, A):=\left(e_{0}(\mathfrak{m} \mid I, A) \ldots, e_{n}(\mathfrak{m} \mid I, A)\right) .
$$

Now suppose we have shown that

$$
e_{*}\left(\mathfrak{m} \mid I\left(a_{1}, \ldots, a_{d}\right)\right)=e_{*}\left(\mathfrak{m} \mid I\left(a_{1}+1, a_{2}, \ldots, a_{d-1}, a_{d}-1\right)\right) .
$$

Then all scrolls of the same dimension and codimension have the same mixed multiplicities $e_{*}(\mathfrak{m} \mid I, A)$. In fact, if $S\left(a_{1}, \ldots, a_{d}\right)$ is not balanced, then we can pass to $S\left(b_{1}, \ldots, b_{d}\right)$, where $\left(b_{1}, \ldots, b_{d}\right)$ is obtained from reordering $\left(a_{1}+\right.$ $\left.1, a_{2}, \ldots, a_{d-1}, a_{d}-1\right)$ to natural order, and we have $e_{*}\left(\mathfrak{m} \mid I\left(a_{1}, \ldots, a_{d}\right)\right)=$ $e_{*}\left(\mathfrak{m} \mid I\left(b_{1}, \ldots, b_{d}\right)\right)$. Repeating this, if necessary, we finally arrive at a balanced scroll with he same mixed multiplicities $e_{*}(\mathfrak{m} \mid I, A)$.

In order to prove (19), we consider the rational normal scroll

$$
S\left(1, a_{1}, \ldots, a_{d-1}, a_{d}-1\right) \subset \mathbb{P}^{n+1}
$$

from which $S\left(a_{1}, \ldots, a_{d}\right)$ and $S\left(a_{1}+1, a_{2}, \ldots, a_{d-1}, a_{d}-1\right)$ can be obtained by intersecting it with suitable hyperplanes. To be more precise, we remember that

$$
J:=I\left(1, a_{1}, \ldots, a_{d-1}, a_{d}-1\right) \subset \tilde{A}:=A\left[x_{n+1}\right]=K\left[x_{0}, \ldots, x_{n+1}\right]
$$


is generated by the 2 -minors of the $2 \times(c+1)$ matrix

$\left(\begin{array}{c|ccc|ccc|c|ccc}x_{0} & x_{2} & \ldots & x_{a_{1}+1} & x_{a_{1}+3} & \ldots & x_{a_{1}+a_{2}+2} & \ldots & x_{n-a_{d}+2} & \ldots & x_{n} \\ x_{1} & x_{3} & \ldots & x_{a_{1}+2} & x_{a_{1}+4} & \ldots & x_{a_{1}+a_{2}+3} & \ldots & x_{n-a_{d}+3} & \ldots & x_{n+1}\end{array}\right)$,

which has $d+1$ blocks. If we merge the first and the last block by setting $x_{1}=x_{n-a_{d}+2}$, we obtain the matrix whose 2 -minors generate $I\left(a_{1}, \ldots, a_{d}\right)$, and if we merge the first and the second block by setting $x_{1}=x_{2}$, we obtain the matrix whose 2 -minors generate $I\left(a_{1}+1, a_{2}, \ldots, a_{d-1}, a_{d}-1\right)$, hence

$$
\begin{aligned}
e_{*}\left(\tilde{\mathfrak{m}} \mid J, \tilde{A} /\left(x_{1}-x_{n-a_{d}+2}\right)\right) & =e_{*}\left(\mathfrak{m} \mid I\left(a_{1}, \ldots, a_{d}\right), A\right) \quad \text { and } \\
e_{*}\left(\tilde{\mathfrak{m}} \mid J, \tilde{A} /\left(x_{1}-x_{2}\right)\right) & =e_{*}\left(\mathfrak{m} \mid I\left(a_{1}+1, a_{2}, \ldots, a_{d-1}, a_{d}-1\right), A\right)
\end{aligned}
$$

Since the mixed multiplicities $e_{k}(\tilde{\mathfrak{m}} \mid J, \tilde{A})$ are the coefficients of the terms of highest degree in the Hilbert polynomial of the bigraded ring Rees algebra $R_{J}(\widetilde{A})$ and the linear forms $x_{1}-x_{n-a_{d}+2}$ and $x_{1}-x_{2}$ are non zero-divisors in the integral domain $R_{J}(\tilde{A})$, we have for $k=0, \ldots, n$ the equalities

$$
e_{k}(\tilde{\mathfrak{m}} \mid J, \tilde{A})=e_{k}\left(\tilde{\mathfrak{m}} \mid J, \tilde{A} /\left(x_{1}-x_{n-a_{d}+2}\right)\right)=e_{k}\left(\tilde{\mathfrak{m}} \mid J, \tilde{A} /\left(x_{1}-x_{2}\right)\right),
$$

see [42, Theorem 5] or [3, Proposition 1.6]. Combining (20), (21) and (22), we get (19), which finishes the proof.

The two preceding theorems give immediately the following result.

Theorem 4.4. Let $S\left(a_{1}, \ldots, a_{d}\right) \subset \mathbb{P}^{n}$ be a d-dimensional rational normal scroll of codimension $c\left(=\sum_{i=1}^{d} a_{i}-1\right)$. Then, for $k=0, \ldots, n$, its degrees of the Segre class and the Stückrad-Vogel cycle (that is, the generalized Samuel multiplicities) are respectively

$$
\begin{aligned}
s^{k}=s_{n-k} & =\sum_{i=0}^{k}\left(\begin{array}{c}
k-1 \\
i-1
\end{array}\right)(-2)^{k-i}(2 c+1-i)\left[\left(\begin{array}{c}
i-1 \\
c-1
\end{array}\right)-\left(\begin{array}{c}
i-1 \\
c
\end{array}\right)\right], \\
c_{0}(I, A) & =2 e_{n}(\mathfrak{m} \mid I)=2 \sum_{i=d}^{c}\left(\begin{array}{c}
n \\
i
\end{array}\right)-2(c-d+1)\left(\begin{array}{c}
n \\
c
\end{array}\right) \\
c_{n+1-k}(I, A) & =(2 c+1-k)\left[\left(\begin{array}{c}
k-1 \\
c-1
\end{array}\right)-\left(\begin{array}{c}
k-1 \\
c
\end{array}\right)\right]
\end{aligned}
$$

\section{References}

[1] R. Achilles, D. Aliffi, Segre, a script for the REDUCE package CALI Bologna, 1999-2017, http://www.dm.unibo.it/ achilles/segre/.

[2] R. Achilles, M. Manaresi, Multiplicity for ideals of maximal analytic spread and intersection theory, J. Math. Kyoto Univ. 33 (1993) 1029-1046.

[3] R. Achilles, M. Manaresi, Multiplicities of a bigraded ring and intersection theory, Math. Ann. 309 (1997) 573-591. 
[4] P. Aluffi, Computing characteristic classes of projective schemes, J. Symbolic Comput. 35 (2003) 3-19.

[5] P. B. Bhattacharya, The Hilbert function of two ideals, Proc. Cambridge Philos. Soc. 53 (1957) 568-575.

[6] C. Biviá-Ausina, Joint reductions of monomial ideals and multiplicity of complex analytic maps, Math. Res. Lett. 15 (2008) 389-407.

[7] E. Bod'a, W. Vogel, On system of parameters, local intersection multiplicity and Bezout's theorem, Proc. Amer. Math. Soc. 78 (1980) 1-7.

[8] W. Bruns, J. Herzog, Cohen-Macaulay rings (Cambridge Studies in Advanced Mathematics, 39. Cambridge University Press, Cambridge, 1993).

[9] C. Ciupercă, A numerical characterization of the $S_{2}$-ification of a Rees algebra, J. Pure Appl. Algebra 178 (2003) 25-48.

[10] A. Conca, J. Herzog, G. Valla, Sagbi bases with applications to blow-up algebras, J. Reine Angew. Math. 474 (1996) 113-138.

[11] D. Eklund, C. Jost, C. Peterson, A method to compute Segre classes of subschemes of projective space, J. Algebra Appl. 12 (2013) 125-142.

[12] H. Flenner, M. Manaresi, A length formula for the multiplicity of distinguished components of intersections, J. Pure Appl. Algebra 165 (2001) $155-168$.

[13] H. Flenner, L. O'Carroll, W. Vogel, Joins and intersections (Springer Monographs in Mathematics. Springer-Verlag, Berlin, 1999).

[14] W. Fulton, Intersection theory (Second edition. Ergebnisse der Mathematik und ihrer Grenzgebiete, Folge 3, Vol. 2. Springer-Verlag, Berlin, 1998).

[15] T. Gaffney, R. Gassler, Segre numbers and hypersurface singularities, J. Algebraic Geom. 8 (1999) 695-736.

[16] L. J. van Gastel, Excess intersections and a correspondence principle, Invent. Math. 103 (1991) 197-222.

[17] C. Harris, Computing Segre classes in arbitrary projective varieties, J. Symbolic Comput. 82 (2017) 26-37.

[18] J. Harris, Algebraic geometry. A first course. Corrected reprint of the 1992 original (Graduate Texts in Mathematics, 133. Springer-Verlag, New York, 1995).

[19] M. Helmer, Algorithms to Compute Characteristic Classes (2015) (Electronic Thesis and Dissertation Repository. 2923. http://ir.lib.uwo.ca/etd/2923). 
[20] M. Helmer, Algorithms to compute the topological Euler characteristic, Chern-Schwartz-MacPherson class and Segre class of projective varieties, J. Symbolic Comput. 73 (2016) 120-138.

[21] M. Herrmann, E. Hyry, J. Ribbe, Z. Tang, Reduction numbers and multiplicities of multigraded structures, J. Algebra 197 (1997) 311-341.

[22] M. Herrmann, S. Ikeda, U. Orbanz, Equimultiplicity and blowing up. An algebraic study. With an appendix by B. Moonen (Springer-Verlag, Berlin, 1988).

[23] N. D. Hoang, H. M. Lam, Mixed multiplicities of rational normal scrolls, Comm. Algebra 40 (2012) 4588-4603.

[24] C. Huneke, I. Swanson, Integral closure of ideals, rings, and modules (London Mathematical Society Lecture Note Series, 336. Cambridge University Press, Cambridge, 2006).

[25] J. Jeffries, J. Montaño, M. Varbaro, Multiplicities of classical varieties, Proc. Lond. Math. Soc. (3) 110 (2015) 1033-1055.

[26] D. Katz, J. K. Verma, Extended Rees algebras and mixed multiplicities, Math. Z. 202 (1989) 111-128.

[27] D. B. Massey, The Lê varieties. I, Invent. Math. 99 (1990) 357-376.

[28] D. B. Massey, The Lê varieties. II, Invent. Math. 104 (1991) 113-148.

[29] H. Matsumura, Commutative algebra (Second edition, Mathematics Lecture Note Series, 56. Benjamin/Cummings Publishing Co., Inc., Reading, Mass., 1980).

[30] M. Nagata, Local rings (Corrected reprint. Robert E. Krieger Publishing Co., Huntington, N.Y., 1975).

[31] D. G. Northcott, D. Rees, Reductions of ideals in local rings, Proc. Cambridge Philos. Soc. 50 (1954) 145-158.

[32] D. Rees, a-transforms of local rings and a theorem on multiplicities of ideals, Proc. Cambridge Philos. Soc. 57 (1961) 8-17.

[33] D. Rees, Generalizations of reductions and mixed multiplicities, J. London Math. Soc. (2) 29 (1984) 397-414.

[34] J. Stückrad, W. Vogel, An algebraic approach to the intersection theory, (The curves seminar at Queens, Vol. II (Kingston, Ont., 1981/1982), Exp. No. A, 32 pp., Queen's Papers in Pure and Appl. Math., 61, Queen's Univ., Kingston, ON, 1982). 
[35] B. Teissier, Cycles évanescents, sections planes et conditions de Whitney, Singularités à Cargèse (Rencontre Singularités Géom. Anal., Inst. Études Sci., Cargèse, 1972), pp. 285-362. Astérisque, Nos. 7 et 8, Soc. Math. France, Paris, 1973.

[36] N. V. Trung, Positivity of mixed multiplicities, Math. Ann. 319 (2001) $33-63$.

[37] N. V. Trung, J. K. Verma, Hilbert functions of multigraded algebras, mixed multiplicities of ideals and their applications, J. Commut. Algebra 2 (2010) $515-565$.

[38] P. Tworzewski, Intersection theory in complex analytic geometry, Ann. Polon. Math. 62 (1995) 177-191.

[39] J. K. Verma, Rees algebras and mixed multiplicities, Proc. Amer. Math. Soc. 104 (1988) 1036-1044.

[40] J. K. Verma, Multigraded Rees algebras and mixed multiplicities, J. Pure Appl. Algebra 77 (1992) 219-228.

[41] W. Vogel, Lectures on results on Bezout's theorem. Notes by D. P. Patil (Tata Institute of Fundamental Research Lectures of Mathematics and Physics, 74. Published for the Tata Institute of Fundamental Research, Bombay, by Springer-Verlag, Berlin-Heidelberg-New York, 1984).

[42] B. L. van der Waerden, On Hilbert's function, series of composition of ideals and a generalisation of the theorem of Bezout, Proc. Roy. Acad. Amsterdam 31 (1929) 749-770. 